\title{
Reproducibility of Retinal Nerve Fiber Layer Thickness Measurements Using Spectral Domain Optical Coherence Tomography
}

\author{
Huijuan Wu, M.D., Ph.D. ${ }^{1,2}$, Johannes F. de Boer, Ph.D. ${ }^{3}$, and Teresa C. Chen, M.D. ${ }^{1}$ \\ ${ }^{1}$ Glaucoma Service, Massachusetts Eye and Ear Infirmary, Department of Ophthalmology, \\ Harvard Medical School, Boston, Massachusetts ${ }^{2}$ Department of Ophthalmology, Peking \\ University, People's Hospital, Beijing, China ${ }^{3}$ Department of Physics and Astronomy, VU \\ University, Amsterdam, The Netherlands
}

\begin{abstract}
Purpose-To evaluate the reproducibility of peri-papillary retinal nerve fiber layer (RNFL) thickness measurements obtained by Spectralis spectral domain optical coherence tomography (OCT) (Heidelberg Engineering, Heidelberg, Germany) in normal and glaucoma subjects.

Methods-Participants were recruited from a university-based clinic. Peri-papillary RNFL thickness measurements were repeated three times during the same visit using the follow-up function. One eye of each participant was randomly selected for statistical analysis.

Reproducibility was evaluated using within-subject standard deviation ( $\mathrm{Sw}$ ), coefficient of variation (CV), and intraclass correlation coefficient (ICC). Spearman's rank correlation coefficient analyses were used to assess the correlation of the standard deviation of the 3 measurements for each participant with the RNFL thickness value.
\end{abstract}

Results-Forty-five normal subjects and 33 glaucoma patients were included in the study. The CVs ranged from $1.45 \%$ [overall global $(\mathrm{G})$ ] to $2.59 \%$ [temporal quadrant $(\mathrm{T})$ ] in normal eyes and from $1.74 \%(\mathrm{G})$ to $3.22 \%(\mathrm{~T})$ in glaucomatous eyes. ICCs ranged from 0.977 (T) to 0.990 (G and inferior-nasal sector) in normal eyes and from 0.983 (T) to 0.997 (inferior quadrant) in glaucomatous eyes. Sw were from $1.34 \mu \mathrm{m}(\mathrm{G})$ to $2.39 \mu \mathrm{m}$ (superior-temporal and inferiortemporal sectors) in normal eyes and from $1.14 \mu \mathrm{m}(\mathrm{G})$ to $2.25 \mu \mathrm{m}$ (superior-nasal sector) in glaucomatous eyes. There were no significant correlations between RNFL thickness values and measurement variability for each participant.

Conclusions-Spectralis OCT shows excellent reproducibility for measuring peri-papillary RNFL thickness values in both healthy and glaucoma subjects.

\section{Keywords}

optical coherence tomography; retinal nerve fiber layer; reproducibility; glaucoma

Correspondence to: Teresa C. Chen, M.D. Mailing address: Massachusetts Eye and Ear Infirmary Glaucoma Service 243 Charles Street, Boston, MA, 02114 teresa_chen @ meei.harvard.edu Telephone number: 617-573-3674 Fax number: 617-573-4300.

This is a PDF file of an unedited manuscript that has been accepted for publication. As a service to our customers we are providing this early version of the manuscript. The manuscript will undergo copyediting, typesetting, and review of the resulting proof before it is published in its final citable form. Please note that during the production process errors may be discovered which could affect the content, and all legal disclaimers that apply to the journal pertain. 


\section{Introduction}

Optical coherence tomography (OCT), first described by Huang et al in $1991,{ }^{1}$ allows for noninvasive in vivo cross-sectional imaging of ocular structures such as the retina, retinal nerve fiber layer (RNFL), and optic nerve head. ${ }^{1-21}$ OCT applies the principle of interferometry to determine the interface between different ocular tissues. ${ }^{1-5}$ Using automated segmentation algorithms based on reflectivity changes between adjacent retinal layers, the RNFL thickness can be calculated. ${ }^{2,4,5,21}$

Before the end of 2006, the OCT instruments used in clinical practice for RNFL thickness determination were based on time domain OCT (TD-OCT) technology. ${ }^{6-13,20}$ In TD-OCT, images are acquired by evaluating the interference pattern created by the echo time delays of backscattered light from the subject's retina and those from a moving reference mirror. ${ }^{1,2,5,19,20}$ Stratus OCT (Carl Zeiss Meditec, Dublin, CA) is the leading commercially available TD-OCT machine ${ }^{10-13,20}$ and can quantify the RNFL thickness at an axial resolution of about $10 \mu \mathrm{m}$ with a scan speed of $400 \mathrm{~A}$-lines per second. The main impediments to TD-OCT technology are the limited resolution and slow acquisition speed. ${ }^{4}$

Spectral domain OCT (SD-OCT), ${ }^{21-25}$ commercially available in the United States in late 2006, is a newer technology which affords unprecedented ultra-high resolution ultra-high speed RNFL imaging. ${ }^{4,5,14-20}$ This technology is possible, because it uses a spectrometer which is a fundamentally more efficient way to process information coming back from the reference mirror and the eye. ${ }^{20,26,27}$ However, before any new imaging instrument can be widely accepted for the longitudinal evaluation of glaucoma patients, both its reproducibility and diagnostic accuracy must be evaluated. Reproducibility data is particularly important in the longitudinal evaluation of a glaucoma patient undergoing progressive RNFL thinning. Detectable RNFL thinning requires a change greater than that expected for measurement reproducibility or variation. The reproducibility of different SD-OCT devices has been evaluated recently. ${ }^{14-17}$

The Spectralis OCT has a scan speed of 40,000 A-lines per second and an eye tracking system, the TruTrack ${ }^{\mathrm{TM}}$ image alignment software, which enables real-time simultaneous imaging and eye movement tracking. Utilizing the TruTrack ${ }^{\mathrm{TM}}$ image alignment software, the Spectralis OCT is able to continuously monitor the position of the eye. Once eye tracking is engaged, the Spectralis OCT tracks the motion of the eye and can direct the OCT beam to the correct position. Applying the eye tracking technology, multiple images are obtained from exactly the same location and then these images are combined to reduce speckle noise. TruTrack ${ }^{\mathrm{TM}}$ can also precisely track change over time by automatically looking for the same area on the retina. After selecting any prior scan as a reference scan, the Spectralis OCT can align the reference fundus image with the patient's current fundus image at a follow-up visit. The eye tracker recognizes the retina and then directs the current scan to the same location as the reference scan. This technique eliminates subjective or inaccurate scan placement by the operator.

To our knowledge, the current study is the first study designed to evaluate the reproducibility of Spectralis OCT by determining the within-subject standard deviation $(\mathrm{Sw})$, coefficient of variation $(\mathrm{CV})$ and intraclass correlation coefficient (ICC) of peripapillary RNFL thickness measurements in normal subjects and glaucoma patients. 


\section{Methods}

\section{Study Participants and Exam}

All study subjects were recruited consecutively from the glaucoma service at the Massachusetts Eye and Ear Infirmary (MEEI) between April 2009 to July 2009. Each patient had a complete ophthalmologic examination, including history, visual acuity (VA) testing, refractive error exam, Goldmann applanation tonometry, slit-lamp biomicroscopy, gonioscopy, central corneal thickness by ultrasonic pachymetry, dilated ophthalmoscopy of the posterior segment, visual field (VF) testing [Swedish Interactive Threshold Algorithm (SITA) 24-2 test of the Humphrey visual field analyzer 750i, Carl Zeiss Meditec, Dublin, CA], and peri-papillary RNFL thickness measurements using the Spectralis OCT (Spectralis software version 4.0). Inclusion criteria included patients with a spherical equivalent between -5.0 diopters and +5.0 diopters and with best corrected VA of 20/40 or better. The study included only patients with reliable VF testing with acceptable fixation losses as defined by the Ocular Hypertension Treatment Study (OHTS) of less than 33\% fixation losses. ${ }^{28}$ Patients also had to have had less than $20 \%$ false positives and $20 \%$ false negatives. Exclusion criteria included patients with 1) any anterior segment dysgenesis, 2) corneal scarring or opacities, 3) proliferative or non-proliferative diabetic retinopathy, 4) field loss attributable to a non-glaucoma condition, 5) a dilated pupil diameter of less than 2 $\mathrm{mm}$.

Participants included normal subjects and glaucoma patients. Normal subjects were those without significant ocular disease (except for mild cataract) and with normal VF test results that were defined as a pattern standard deviation (PSD) $>5 \%$ and glaucoma hemifield test (GHT) within normal limits. ${ }^{28}$ Glaucoma patients included those with any form of chronic glaucoma except for traumatic glaucoma and were defined as having characteristic glaucomatous disc abnormality with corresponding VF loss. The VF was considered abnormal if three or more contiguous test locations in the pattern deviation plot were depressed significantly at the $P<5 \%$ level with at least 1 at the $P<1 \%$ level on the same side of the horizontal meridian, or was classified as outside normal limits by the GHT. ${ }^{29}$ If both eyes of one subject fit inclusion and exclusion criteria, one eye was selected randomly by using a random number generator statistical table.

\section{Spectralis OCT Peri-papillary Retinal Nerve Fiber Layer Thickness Measurement}

All peri-papillary RNFL thickness measurements were made with the Spectralis OCT using a circular scan pattern (Spectralis software version 4.0). The scan circle was 12 degrees in diameter, and the diameter in millimeter is primarily dependent on the axial eye length. For a typical eye length, the circle is approximately $3.5-3.6 \mathrm{~mm}$ in diameter.

As described in the introduction section, Spectralis OCT provides an Automatic Real-Time (ART) function which utilizes TruTrack ${ }^{\mathrm{TM}}$ image alignment software (i.e. the eye tracking system). With ART activated, multiple frames of the same scanning location are performed during the scanning process, and images are averaged for speckle noise reduction. In this study where ART was activated, 16 frames were acquired and then averaged for each scan. In addition, the Spectralis OCT eye tracking system allows any subsequent OCT scan to be scanned at exactly the same location as a prior scan. For subjects in the current study, a circular peri-papillary scan was acquired first and then defined as a reference scan (first scan). Then the camera was re-started, the follow-up button in the acquisition window was pressed, a previous reference image was selected, and then another circular peri-papillary scan (second scan, Figure 1) at the same location as the reference image would be obtained. This follow-up function was then repeated for the third image (third scan). By this method, three circular scans of the peri-papillary RNFL were obtained at exactly the same location. 
The Spectralis OCT software calculates the average RNFL thickness for the overall global (360 degrees), for 4 quadrants (superior [S], inferior [I], nasal [N], and temporal [T], each 90 degrees), and then for four additional sectors (i.e. superior-temporal [TS, $45^{\circ}-90^{\circ}$ ], superiornasal [NS, $90^{\circ}-135^{\circ}$ ], inferior-nasal [NI, $225^{\circ}-270^{\circ}$ ], and inferior-temporal [TI, $270^{\circ}$ $\left.-315^{\circ}\right]$ ). The signal strength (range, $0-40$ ) of each scan was reviewed, and scans with signal strength of less than 15 (as suggested by the manufacturer) were excluded from the analysis. In addition, criteria for determining scan quality were modified after previous studies ${ }^{11,15,17}$ and included the following: a clear fundus image allowing visualization of the optic disc and the scan circle before and during image acquisition, RNFL visible and without interruptions, and a continuous scan pattern without missing or blank areas.

\section{Statistical Analysis}

Demographic and ocular characteristics of the normal and glaucoma groups were compared using chi-square tests and non-paired 2-tailed Student's $t$ tests for categorical and continuous variables respectively. The reproducibility of Spectralis OCT RNFL thickness measurements was assessed by calculating the $\mathrm{Sw}$, the $\mathrm{CV}$, and the ICC. The values were determined for each of the RNFL parameters (i.e. overall global, 4 quadrants [S, T, I, and N], and 4 sectors [TS, TI, NS, and NI). For the Sw determination, we used the method that was first described by Bland and Altman ${ }^{30}$ and which has been used in previous studies for assessing reproducibility. ${ }^{15,17}$ The $\mathrm{Sw}$, or within-subject standard deviation, is the common standard deviation of the repeated measurements. In order to calculate the common Sw, we averaged the variances, or the squares of the standard deviations, of all study participants. The square root of the mean within-subject variance is the $\mathrm{Sw}$. CV is a ratio of the standard deviation over the mean and was calculated as the square root of the residual mean squared values of 3 measures, divided by the mean. An SPSS (SPSS, Inc., Chicago, IL) Reliability Analysis using a one-way random model was used to determine ICC. ${ }^{31}$ Spearman's rank correlation coefficient analyses were used to assess the correlation of the standard deviation of the 3 repeated measures for each participant with the specific RNFL thickness measurement. Statistical analyses were performed using SPSS version 15.0 and JMP software version 7.0 (SAS Institute, Cary, North Carolina). $P<0.05$ was considered to be statistically significant, and $P<0.001$ was considered to be highly statistically significant.

\section{Results}

A total of 78 subjects ( 45 normal subjects and 33 glaucoma patients) were included in the study. Table 1 shows the demographic and ocular characteristics of the study population. There were no significant differences between the normal and glaucoma groups in terms of number of right eyes, age, gender, race, and refractive error. There were statistically significant differences in VF mean deviation (MD) $(P<0.001, t$ test $)$ and pattern standard deviation (PSD) $(P<0.001, t$ test) between normal subjects and glaucoma patients. Types of glaucoma included primary open angle glaucoma (23 of 33 patients, $69.7 \%$ ), normal tension glaucoma ( 3 of 33 patients, 9.1\%), pseudoexfoliation glaucoma ( 2 of 33 patients, $6.1 \%$ ), chronic angle closure glaucoma ( 3 of 33 patients, 9.1\%), uveitic glaucoma ( 1 of 33 patients, $3.0 \%$ ), and juvenile open angle glaucoma ( 1 of 33 patients, $3.0 \%$ ).

Table 2 shows the peri-papillary RNFL thickness values of normal subjects and glaucoma patients for overall global, quadrant, and sector RNFL thickness. The overall global RNFL thicknesses values were higher for normal subjects at $92.7 \pm 7.2 \mu \mathrm{m}$ compared to $65.2 \pm$ $18.2 \mu \mathrm{m}$ for glaucoma patients. Mean RNFL thickness values at all locations were significantly thinner (at the $P<0.001$ level) in glaucoma patients compared to normal subjects. 
Table 3 shows the reproducibility of Spectralis OCT peri-papillary RNFL thickness measurements for all study patients. CVs ranged from $1.45 \%$ (overall global) to $2.59 \%$ (temporal quadrant) for normal participants and ranged from $1.74 \%$ (overall global) to $3.22 \%$ (temporal quadrant) for glaucoma patients. ICCs were from 0.977 (temporal quadrant) to 0.990 (overall global and NI sector) in normal eyes and from 0.983 (temporal quadrant) to 0.997 (inferior quadrant) in glaucomatous eyes. Sw \pm 1.96 standard error (se) ranged from $1.34 \pm 0.20 \mu \mathrm{m}$ (overall global) to $2.39 \pm 0.35 \mu \mathrm{m}$ (TS sector and TI sector) for normal subjects and ranged from $1.14 \pm 0.19 \mu \mathrm{m}$ (overall global) to $2.25 \pm 0.38 \mu \mathrm{m}$ (NS sector) for glaucoma patients.

In Figure 2, the overall RNFL thickness values for all subjects were plotted against the standard deviation (SD) of the 3 repeated measures. Figure 2 shows that within-subject variability was unrelated to RNFL thickness (Spearman: $r=-0.063, P=0.582$ ). Table 4 shows all the results (i.e. Spearman $r$ values and $P$ values) of Spearman's rank correlation coefficient analyses between RNFL thickness in all locations and the relevant SD for normal subjects, glaucoma patients, and all participants respectively. There were no statistically significant correlations between RNFL thickness and within-subject variability in any location for all the groups (none of the $P$ values $<0.05$ ).

\section{Discussion}

Assessing an instrument's reproducibility is always the first step in the evaluation of a new technology for use in clinical practice, because reproducibility affects diagnostic accuracy as well as the ability to monitor disease progression. ${ }^{16}$ The current study demonstrates that Spectralis spectral domain OCT peri-papillary RNFL thickness measurements are highly repeatable (ICCs ranging from 0.977 to 0.997 , Table 3) and reproducible (Sw ranging from $1.14 \mu \mathrm{m}$ to $2.39 \mu \mathrm{m}$, Table 3 ) in both normal subjects and glaucoma patients.

Several earlier studies have shown that TD-OCT RNFL thickness measurements are reproducible ${ }^{6-13}$ but more variable in glaucomatous eyes. ${ }^{6,7,11}$ However, TD-OCT technology is inadequate for longitudinal RNFL thickness evaluations, because the testretest variability of TD-OCT RNFL thickness measurements range from 3.5-20.6 $\mu \mathrm{m}$ in normal and glaucoma eyes, ${ }^{12,13}$ which is greater than clinically significant degrees of glaucomatous RNFL thinning. Studies have shown that reductions of RNFL thickness by $3.77 \mu \mathrm{m}$ in monkeys ${ }^{32}$ and $4.3 \mu \mathrm{m}$ in humans ${ }^{33}$ are clinically significant glaucomatous structural changes. In the latter study, OCT RNFL thinning of $4.3 \mu \mathrm{m}$ was associated with clearly observed progressive RNFL atrophy in red-free RNFL photographs. ${ }^{33}$ This means that TD-OCT can not differentiate between a $4 \mu \mathrm{m}$ decrease due to measurement variation versus $4 \mu \mathrm{m}$ of RNFL thinning due to glaucomatous disease progression. In the current study, the Sw of Spectralis OCT ranges from 1.14-2.39 $\mu \mathrm{m}$, which is less than what is considered significant glaucomatous structural change $(4.3 \mu \mathrm{m})$. Thus, Spectralis OCT RNFL thickness measurements have better potential than TD-OCT in the longitudinal evaluation of glaucomatous RNFL thinning.

Studies in the literature have shown that the reproducibility values (i.e. ICC) for global and quadrant peri-papillary RNFL thickness measurements using the commercially available SD-OCT machines (e.g. Cirrus HD-OCT [Carl Zeiss Meditec, Inc., Dublin, CA], 3D OCT-1000 [Topcon, Tokyo, Japan], and RTVue OCT [Optovue Corporation, Freemont, CA]) range from 0.742 to $0.994,{ }^{14-18}$ which are worse than the ICC values of 0.977 to 0.997 for the Spectralis machine (Table 3). Therefore, although the reproducibility of RNFL measurements for the commercially available SD-OCTs are all excellent, the Spectralis SDOCT system appears to have the best reproducibility, based on the results of the aforementioned studies as well as results from the current study. Studies of SD-OCT RNFL 
reproducibility using Sw show similar results, with Cirrus and RTVue RNFL thickness measurement having more variability (i.e. Sw 1.2-2.9 $\mu \mathrm{m}$ for Cirrus ${ }^{17}$ and $1.57-3.67 \mu \mathrm{m}$ for RTVue $^{15}$ ) compared to Spectralis RNFL measurements (Sw: 1.14-1.94 $\mu \mathrm{m}$, Table 3). The superiority of Spectralis SD-OCT's reproducibility has also been suggested in a recent study of 20 healthy subjects where macular thickness values were determined by six commercially available OCT instruments, 5 of which were SD-OCT machines: Spectralis OCT, Cirrus HD-OCT, OCT/SLO [Opko/OTI, Inc., Miami, FL], RTVue-100 OCT, SOCT [Reichert/ Optopol Technology, Inc., Depew, NY], and Stratus OCT. ${ }^{3}$ In that study, the Spectralis OCT showed the lowest CV $(0.46 \%)$ compared to any other SD-OCT machine (i.e. 3.09\% for the Cirrus HD-OCT, $2.23 \%$ for the OCT/SLO, $2.77 \%$ for the RTVue-100 OCT, 3.5\% for the SOCT) and to the leading TD-OCT machine (3.33\% for Stratus OCT), ${ }^{34}$ which suggests that Spectralis OCT may have the best reproducibility among all commercially available OCT machines.

The current study using Spectralis SD-OCT confirm two main findings from the previous reproducibility OCT studies. First, when using SD-OCT technology, the reproducibility for peri-papillary RNFL thickness measurements are equally excellent in both normal and glaucoma eyes for RTVue OCT ${ }^{15}$ and Cirrus HD-OCT, ${ }^{17}$ which is in contrast to TD-OCT studies that note more variability in RNFL measurements with glaucoma eyes. ${ }^{6,7,11}$ Similarly in this Spectralis SD-OCT study (Table 3), RNFL reproducibility was similar for glaucoma eyes (ICC range 0.983 - 0.997) and normal eyes (ICC range $0.977-0.990$ ). Second, in both TD-OCT and SD-OCT studies, nasal and temporal quadrant measurements generally have more variability and higher CVs compared to superior and inferior quadrant measurements. . $11,12,14-17$ In the current SD-OCT study (Table 3), measurement variability initially appears greater in the nasal and temporal quadrants for both normal and glaucoma eyes (CVs ranged from $2.31 \%-3.22 \%$ ) compared to the superior and inferior quadrants in the same groups (CVs ranged from $1.66 \%-2.30 \%$ ). However in terms of Sw and ICC (Table 3), we could not find consistently higher Sw or lower ICCs in the nasal or temporal quadrants compared to the superior and inferior quadrants. Thus, from the results of this study, we can not definitively conclude that the nasal or temporal quadrants consistently provide the lowest reproducibility among the four quadrants for Spectralis SD-OCT RNFL measurements. One possible reason for the many studies that demonstrate higher CVs in the nasal and/or temporal quadrants may be due to normal anatomy, in that the nasal and temporal RNFL are usually thinner than the superior and inferior RNFL. Since CV is calculated as the SD divided by the mean RNFL thickness and if SD values are similar in different quadrants, the normally thinner nasal and temporal quadrants would usually have higher CV values. Therefore, other statistical values, such as Sw and ICC, should be considered together with $\mathrm{CV}$ for assessing reproducibility.

The findings of the current study are similar to that reported by Budenz et al, ${ }^{12}$ who observed that the actual RNFL thickness value does not seem to have an impact on the variability of the measurement. In Budenz's study of Stratus TD-OCT in 51 eyes of 51 glaucoma patients using the Standard and Fast scan protocols, he evaluated the correlation between RNFL thickness and intersession variability (i.e. scans were obtained on 5 different days within a 2-month period). ${ }^{12}$ This study compared the mean RNFL thickness with the $\mathrm{SD}$ of the 5 repeated measures, and they found no statistical correlation between intersession variability and mean RNFL thickness for both Standard and Fast protocols (Standard: Spearman $r=-0.15, P=0.28$; Fast: Spearman $r=-0.01, P=0.93) .{ }^{12}$ In the current study (Table 4), the correlation coefficients between the mean RNFL thickness values and the SDs of the three repeat measurements were calculated for normal subjects, glaucoma patients and all participants respectively. There was no statistically significant correlation between standard deviation and RNFL thickness values in all locations for all groups (Table 4). 
In conclusion, this study shows that Spectralis SD-OCT technology provides excellent reproducibility of peri-papillary RNFL thickness measurements. With the fastest scan speeds, the Spectralis SD-OCT yields some of the best RNFL thickness reproducibility values of all the commercially available SD-OCT machines. ${ }^{34}$ Further studies with longer follow-up are needed in order to evaluate inter-visit variability and to determine if Spectralis OCT can detect small structural changes over time.

\section{Acknowledgments}

This study was supported in part by the National Institutes of Health (R01 EY14975-01).

\section{References}

1. Huang D, Swanson EA, Lin CP, et al. Optical coherence tomography. Science. 1991; 254:11781181. [PubMed: 1957169]

2. Schuman JS, Hee MR, Puliafito CA, et al. Quantification of nerve fiber layer thickness in normal and glaucomatous eyes using optical coherence tomography. Arch Ophthalmol. 1995; 113:586-596. [PubMed: 7748128]

3. Fujimoto JG, Bouma B, Tearney GJ, et al. New technology for high-speed and high-resolution optical coherence tomography. Ann N Y Acad Sci. Feb 9.1998 838:95-107. [PubMed: 9511798]

4. Chen TC, Cense B, Pierce MC, et al. Spectral domain optical coherence tomography: ultrahighspeed, ultrahigh-resolution ophthalmic imaging. Arch Ophthalmol. 2005; 123(12):1715-1720. [PubMed: 16344444]

5. Knight OJ, Chang RT, Feuer WJ, Budenz DL. Comparison of retinal nerve fiber layer measurements using time domain and spectral domain optical coherent tomography. Ophthalmology. Jul; 2009 116(7):1271-1277. [PubMed: 19395086]

6. Schuman JS, Pedut-Kloizman T, Hertzmark E, et al. Reproducibility of nerve fiber layer thickness measurements using optical coherence tomography. Ophthalmology. Nov; 1996 103(11):1889_ 1898. [PubMed: 8942887]

7. Blumenthal EZ, Williams JM, Weinreb RN, et al. Reproducibility of nerve fiber layer thickness measurements by use of optical coherence tomography. Ophthalmology. Dec; 2000 107(12):22782282. [PubMed: 11097610]

8. Jones AL, Sheen NJ, North RV, Morgan JE. The Humphrey optical coherence tomography scanner: quantitative analysis and reproducibility study of the normal human retinal nerve fibre layer. $\mathrm{Br} \mathrm{J}$ Ophthalmol. 2001; 85:673-6777. [PubMed: 11371486]

9. Carpineto P, Ciancaglini M, Zuppardi E, et al. Reliability of nerve fiber layer thickness measurements using optical coherence tomography in normal and glaucomatous eyes. Ophthalmology. 2003; 110:190-195. [PubMed: 12511365]

10. Paunescu LA, Schuman JS, Price LL, et al. Reproducibility of nerve fiber thickness, macular thickness, and optic nerve head measurements using StratusOCT. Invest Ophthalmol Vis Sci. 2004; 45:1716-1724. [PubMed: 15161831]

11. Budenz DL, Chang RT, Huang X, et al. Reproducibility of retinal nerve fiber thickness measurements using the stratus OCT in normal and glaucomatous eyes. Invest Ophthalmol Vis Sci. Jul; 2005 46(7):2440-2443. [PubMed: 15980233]

12. Budenz DL, Fredette MJ, Feuer WJ, Anderson DR. Reproducibility of peripapillary retinal nerve fiber thickness measurements with stratus OCT in glaucomatous eyes. Ophthalmology. Apr; 2008 115(4):661-666.e4. [PubMed: 17706287]

13. Tzamalis A, Kynigopoulos M, Schlote T, Haefliger I. Improved reproducibility of retinal nerve fiber layer thickness measurements with the repeat-scan protocol using the Stratus OCT in normal and glaucomatous eyes. Graefes Arch Clin Exp Ophthalmol. Feb; 2009 247(2):245-252. [PubMed: 18810480]

14. Menke MN, Knecht P, Sturm V, et al. Reproducibility of nerve fiber layer thickness measurements using 3D Fourier-domain OCT. Invest Ophthalmol Vis Sci. Dec; 2008 49(12):5386-5391. Epub 2008 Aug 1. [PubMed: 18676630] 
15. González-García AO, Vizzeri G, Bowd C, et al. Reproducibility of RTVue retinal nerve fiber layer thickness and optic disc measurements and agreement with Stratus optical coherence tomography measurements. Am J Ophthalmol. Jun; 2009 147(6):1067-1074. 1074.e1. Epub 2009 Mar 9. [PubMed: 19268891]

16. Leung CK, Cheung CY, Weinreb RN, et al. Retinal nerve fiber layer imaging with spectral-domain optical coherence tomography: a variability and diagnostic performance study. Ophthalmology. Jul; 2009 116(7):1257-1263. 1263.e1-2. Epub 2009 May 22. [PubMed: 19464061]

17. Vizzeri G, Weinreb RN, Gonzalez-Garcia AO, et al. Agreement between Spectral-Domain and Time-Domain OCT for measuring RNFL thickness. Br J Ophthalmol. Mar 19.2009 [Epub ahead of print].

18. Kim JS, Ishikawa H, Sung KR, et al. Retinal Nerve Fiber Layer Thickness Measurement Reproducibility Improved with Spectral Domain Optical Coherence Tomography. Br J Ophthalmol. May 7.2009 [Epub ahead of print].

19. Schuman JS. Spectral domain optical coherence tomography for glaucoma (an AOS thesis). Trans Am Ophthalmol Soc. 2008; 106:426-458. [PubMed: 19277249]

20. Chen TC, Zeng A, Sun W, Mujat M, de Boer JF. Spectral domain optical coherence tomography and glaucoma. Int Ophthalmol Clin. 2008 Fall;48(4):29-45. [PubMed: 18936635]

21. Mujat M, Chan RC, Cense B, Park BH, Joo C, Akkin T, Chen TC, de Boer JF. Retinal Nerve Fiber Layer Thickness Map Determined from Optical Coherence Tomography Images. Optics Express. 2005; 13(23):9480-91. [PubMed: 19503151]

22. Fercher AF, Hitzenberger CK, Kamp G, El-Zaiat SY. Measurements of intraocular distances by backscattering spectral interferometry. Opt. Comm. 1995; 117:43-48.

23. Wojtkowski M, Leitgeb R, Kowalczyk A, Bajraszewski T, Fercher AF. In vivo human retinal imaging by Fourier domain optical coherence tomography. Journal of Biomedical Optics. 2002; 7(3):457-463. [PubMed: 12175297]

24. Nassif N, Cense B, Park BH, Yun SH, Chen TC, Bouma BE, Tearney GJ, de Boer JF. In vivo human retinal imaging by ultrahigh-speed spectral domain optical coherence tomography. Optics Letters. 2004; 29(5):480-482. [PubMed: 15005199]

25. Nassif N, Cense B, Park BH, Pierce MC, Yun SH, Bouma BE, Tearney GJ, Chen TC, de Boer JF. In vivo high-resolution video-rate spectral-domain optical coherence tomography of the human retina and optic nerve. Optics Express. 2004; 12(3):367-376. [PubMed: 19474832]

26. Leitgeb R, Hitzenberger CK, Fercher AF. Performance of fourier domain vs. time domain optical coherence tomography. Opt. Express. 2003:889-894. [PubMed: 19461802]

27. de Boer JF, Cense B, Park BH, Pierce MC, Tearney GJ, Bouma BE. Improved signal-to-noise ratio in spectral-domain compared with time-domain optical coherence tomography. Optics Letters. 2003; 28(21):2067-2069. [PubMed: 14587817]

28. Gordon MO, Kass MA. Hypertension Treatment Study: design and baseline description of the participants. Arch Ophthalmol. 1999; 117(5):573-83. [PubMed: 10326953]

29. Katz J, Sommer A, Gaasterland DE, Anderson DR. Comparison of analytic algortihms for detecting glaucomatous visual field loss. Arch Ophthalmol. 1991; 109:1684-9. [PubMed: 1841576]

30. Bland JM, Altman DG. Measurement error. BMJ. Jun 29.1996 312(7047):1654. [PubMed: 8664723]

31. McGraw KO, Wong SP. Forming inferences about some intraclass correlation coefficients. Psychological Methods. 1996; 1(1):30-46.

32. Schuman JS, Pedut-Kloizman T, Pakter H, et al. Optical coherence tomography and histologic measurements of nerve fiber layer thickness in normal and glaucomatous monkey eyes. Invest Ophthalmol Vis Sci. 2007; 48(8):3645-54. [PubMed: 17652734]

33. Lee EJ, Kim TW, Park KH, Seong M, Kim H, Kim DM. Ability of Stratus OCT to Detect Progressive Retinal Nerve Layer Atrophy in Glaucoma. Invest Ophthalmol Vis Sci. 2009; 50(2): 662-668. [PubMed: 18824734]

34. Wolf-Schnurrbusch UE, Ceklic L, Brinkmann CK, et al. Macular thickness measurements in healthy eyes using six different optical coherence tomography instruments. Invest Ophthalmol Vis Sci. Jul; 2009 50(7):3432-7. [PubMed: 19234346] 


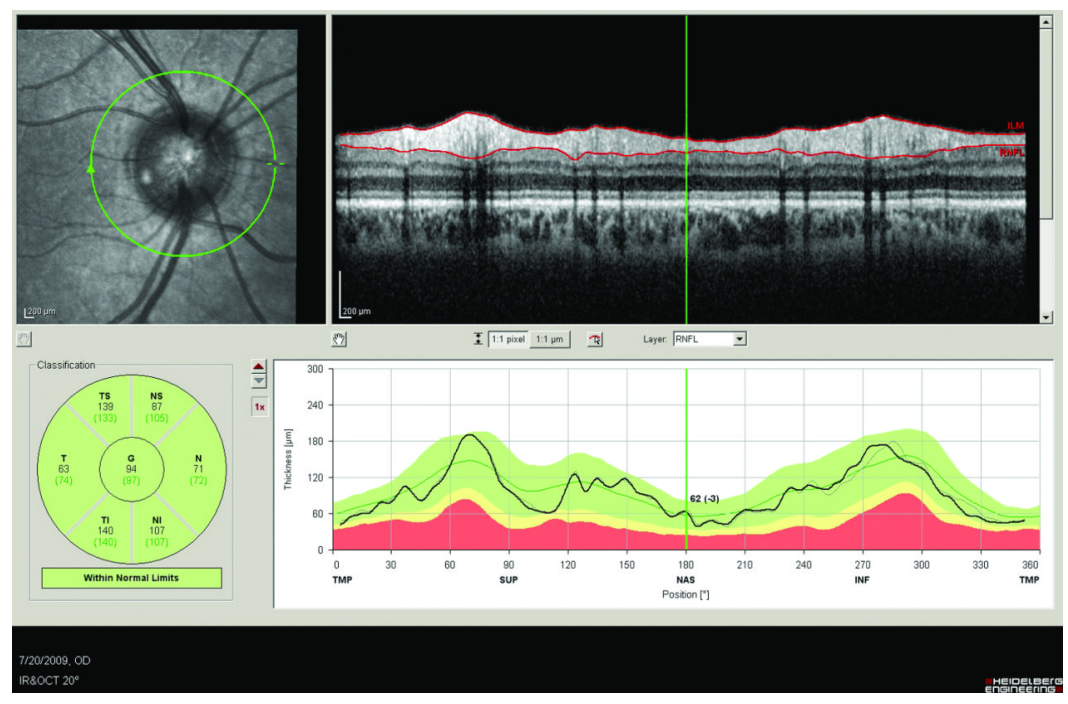

Figure 1.

Example of the second peri-papillary retinal nerve fiber layer (RNFL) circular scan centered on the optic nerve head (ONH) using Spectralis optic coherence tomography (OCT) in a normal eye after applying the follow-up function. The graph on the lower right of the window presents the RNFL thickness detected along the circular scan (black curve), compared to values from the normative database (green curve). The gray curve indicates the values of the chosen reference scan. The black values in the pie chart (lower left) give the average RNFL thickness value for each sector as well as the global average (G).

$\mathrm{N}$ - nasal; T - temporal; TS - superior-temporal; TI - inferior-temporal; NS - superior-nasal; NI - inferior-nasal 


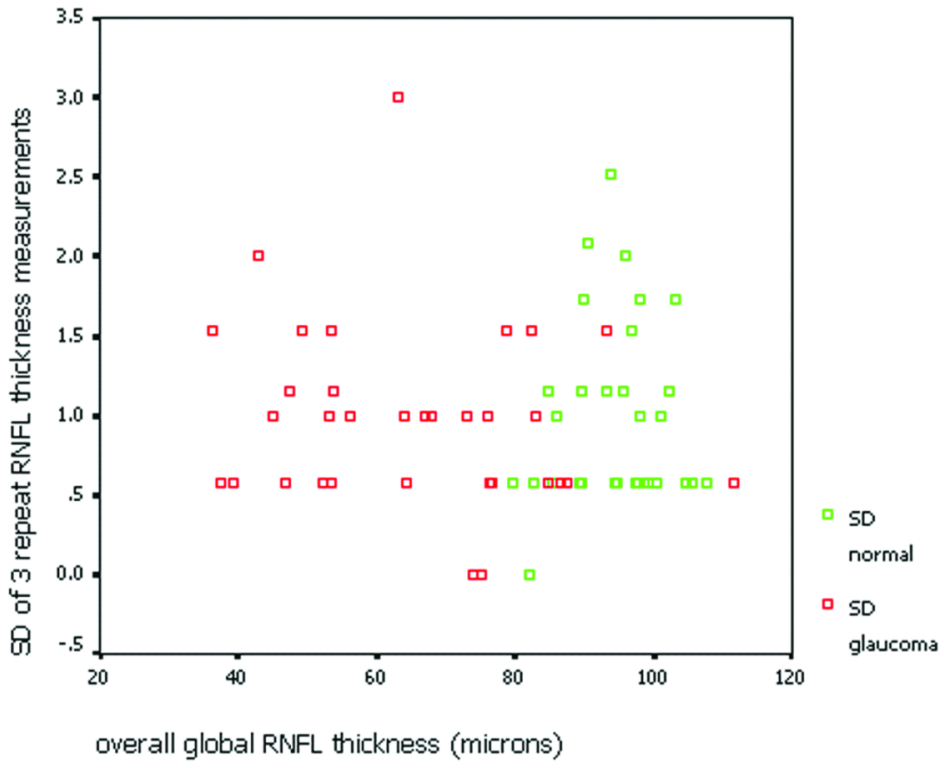

Figure 2.

Figure showing the relationship between the overall global Spectralis optic coherence tomography (OCT) peri-papillary retinal nerve fiber layer (RNFL) thickness measurements versus variability (SD) of 3 repeated measurements. There was no relationship between variability (SD) and overall global RNFL thickness (Spearman $r=-0.063, P=0.582$ ). SD standard deviation. 
Table 1

Patient demographics

\begin{tabular}{llll}
\hline Parameter & normal subjects & glaucoma patients & P value $^{\boldsymbol{a}}$ \\
\hline number of subjects & 45 & 33 & \\
right eyes (\% total) & $69.7 \%$ & $57.8 \%$ & 0.282 \\
Age [mean \pm SD (range) & $66.2 \pm 17.0(20-90)$ & $67.7 \pm 14.2(18-89)$ & 0.057 \\
female (\% total) & $57.8 \%$ & $66.7 \%$ & 0.425 \\
$\begin{array}{l}\text { Race (Caucasian; } \% \text { total) } \\
\text { spherical equivalent in }\end{array}$ & $77.8 \%$ & $66.7 \%$ & 0.274 \\
$\begin{array}{l}\text { diopters (mean } \pm \text { SD) } \\
\begin{array}{l}\text { VF mean deviation in dB } \\
\text { (mean } \pm \text { SD) }\end{array}\end{array}$ & $-1.21 \pm 1.91$ & $-0.98 \pm 2.11$ & 0.357 \\
$\begin{array}{l}\text { VF pattern standard } \\
\text { deviation in dB } \\
\text { (mean } \pm \text { SD) }\end{array}$ & $1.91 \pm 1.70$ & $-11.01 \pm 8.76$ & $<0.001$ \\
\hline
\end{tabular}

VF - visual field; SD - standard deviation

'When comparing normal and glaucoma groups, chi-square tests were used for categorical variables and non-paired 2-tailed Student's $t$ tests was used for continuous variables. 
Table 2

Spectralis optical coherence tomography (OCT) retinal nerve fiber layer (RNFL) thickness measurements $(\mu \mathrm{m})$ in normal and glaucomatous eyes

\begin{tabular}{lcc}
\hline RNFL location & $\begin{array}{c}\text { normal patients }(\mathbf{n}=\mathbf{4 5}) \\
(\text { mean in } \mu \mathbf{m} \pm \mathbf{S D})\end{array}$ & $\begin{array}{c}\text { glaucoma patients }(\mathbf{n}= \\
\text { 33) } \\
\text { (mean in } \mu \mathbf{m} \pm \mathbf{S D})\end{array}$ \\
\hline overall global & $92.7 \pm 7.2$ & $65.2 \pm 18.2^{a}$ \\
superior quadrant & $108.3 \pm 12.4$ & $75.7 \pm 24.9^{a}$ \\
temporal quadrant & $70.4 \pm 12.0$ & $53.7 \pm 13.0^{a}$ \\
inferior quadrant & $117.3 \pm 13.4$ & $73.7 \pm 29.1^{a}$ \\
Nasal quadrant & $73.3 \pm 11.9$ & $57.1 \pm 20.7^{a}$ \\
TS sector & $123.4 \pm 14.5$ & $75.5 \pm 27.5^{a}$ \\
TI sector & $133.9 \pm 17.4$ & $73.3 \pm 27.3^{a}$ \\
NS sector & $94.0 \pm 15.6$ & $71.8 \pm 22.5^{a}$ \\
NI sector & $101.1 \pm 17.8$ & $69.5 \pm 27.3^{a}$ \\
\hline
\end{tabular}

TS - superior-temporal; TI - inferior-temporal; NS - superior-nasal; NI - inferior-nasal

a denotes significantly different $(P<0.001)$ RNFL thickness values when compared to the normal group (analysis of variance) 


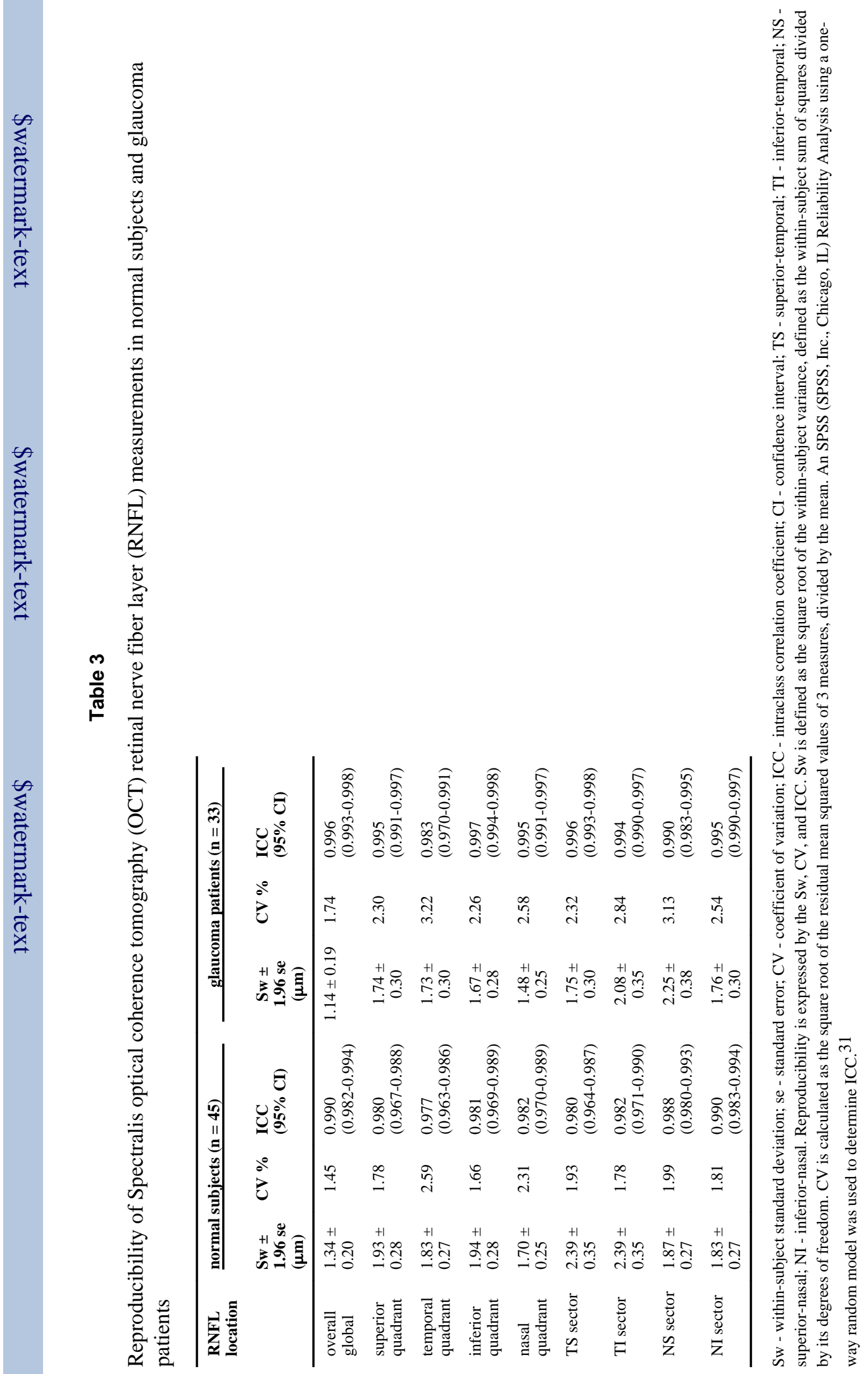




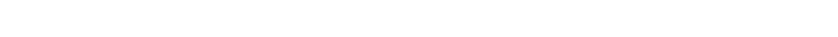

